\title{
CALPOT, fosfato e silício co-aplicados ao solo para a cultura da soja
}

\section{CALPOT, phosphate and silicon co-applied to the soil on soybean crop}

\author{
Cristine Elizabeth Alvarenga Carneiro ${ }^{1 *}$; Roberto Antunes Fioretto ${ }^{2}$; \\ Inês Cristina Batista Fonseca ${ }^{2}$; Geraldo Estevam de Souza Carneiro ${ }^{3}$
}

\section{Resumo}

\begin{abstract}
Visando fornecer $\mathrm{Ca}, \mathrm{Mg}, \mathrm{K}$ e melhorar a disponibilidade de $\mathrm{P}$ para a soja, foi aplicado CALPOT e escória de siderurgia. As fontes também possuem poder de neutralização e diminuição da acidez do solo. Os tratamentos aplicados constituíram-se de: 1) Testemunha; 2) CALPOT + P + Si; 3) CALPOT + P; 4) CALPOT + Si; 5) $\mathrm{Si}+\mathrm{P}$; 6) Calcário; 7) CALPOT. Os cátions básicos $\mathrm{Ca}, \mathrm{Mg}$ e $\mathrm{K}$ foram aplicados como CALPOT, (calcário dolomítico + cloreto de potássio) com dose calculada para se atingir um índice de saturação no solo equivalente a: 50\% de Ca, 10\% de Mg, e 3\% de K. O P aplicado foi de $100 \mathrm{~kg} \mathrm{ha}^{-1} \mathrm{e} \mathrm{Si}$ foi duas vezes a dose de $\mathrm{P}\left(200 \mathrm{~kg} \mathrm{ha}^{-1}\right)$. O tratamento CALPOT $+\mathrm{P}+\mathrm{Si}$ atingiu o equilíbrio em bases como pré-estabelecido, aumentou disponibilidade de fósforo no solo, obteve maior peso de folhas e matéria seca total. A co-aplicação dos elementos não impossibilitou o fornecimento de fósforo e demais nutrientes à planta.
\end{abstract}

Palavras-chave: Cátions básicos, equilíbrio químico, nutrição, fósforo

\begin{abstract}
Seeking to supply $\mathrm{Ca}, \mathrm{Mg}, \mathrm{K}$ and to improve the $\mathrm{P}$ availability to soybean, CALPOT and metallurgy scum were applied. The treatments were: 1) Control; 2) CALPOT $+\mathrm{P}+\mathrm{Si}$; 3) CALPOT $+\mathrm{P}$; 4) CALPOT $+\mathrm{Si} ; 5$ ) $\mathrm{Si}+\mathrm{P}$; 6) Lime; 7) CALPOT. The basic cátions $\mathrm{Ca}, \mathrm{Mg}$ and $\mathrm{K}$ were applied as CALPOT, (dolomitic lime and potassium cloride) with dosages in order to reach the base equilibrium of: $50 \% \mathrm{Ca}, 10 \% \mathrm{Mg}$, and $3 \%$ K. The applied P was $100 \mathrm{~kg} \mathrm{ha}^{-1}$ and Si twice of P dose $\left(200 \mathrm{~kg} \mathrm{ha}^{-1}\right)$. The CALPOT $+\mathrm{P}+\mathrm{Si}$ treatments established the balance in bases, incrased the $\mathrm{P}$ availability in the soil, and produced larger weight of leaves, and total dry matter. The co-application of these elements did not disable phosphorus supply and other nutrients to the plants.
\end{abstract}

Key words: Index terms, basic cátions, chemical balance, nutrition, phosphorus

1 Bacharel e Licenciada em Química, Mestre em Agronomia. Aluna do Programa de Pós-graduação em Agronomia - UEL. LondrinaPR.E-mail: cristine@uel.br.

2 Professores do Departamento de Agronomia, UEL, Londrina, PR.

3 Pesquisador da Embrapa Soja. Londrina -PR. E-mail: estevam@cnpso.embrapa.br

* Autor para correspondência. 


\section{Introdução}

Os fatores que influenciam o crescimento vegetal são genéticos e ambientais, sendo os fatores ambientais um conjunto de condições externas que afetam a vida e o desenvolvimento de uma planta. $\mathrm{O}$ suprimento de nutrientes, por sua vez, depende, entre outros fatores, das características do solo.

Freqüentemente o estado de baixa fertilidade dos solos agricultáveis é caracterizado pela elevada acidez e baixo teor de nutrientes como $\mathrm{Ca}, \mathrm{Mg}, \mathrm{K}$ e $\mathrm{P}$, assim como, solos contendo apreciáveis quantidades de alumínio e ferro, são altamente responsáveis pela adsorção de fosfatos, os quais formam compostos insolúveis (PARFITT, 1978) que por sua vez tornam o fósforo indisponível. Na literatura são encontrados vários estudos que possibilitam melhorar a disponibilidade de P nos solos (MUNIZ et al.,1985; CASAGRANDE; CAMARGO,1997; LEITE, 1997; MOTTA et al., 2002, CARNEIRO, 2003), sendo a aplicação de silicatos uma delas. A correção da acidez do solo com silicatos, além de elevar o $\mathrm{pH}$, pode disponibilizar o $\mathrm{P}$, pelo efeito adicional de deslocar o P adsorvido para a solução (VOLKWEISS; RAIJ, 1976). A interação silíciofósforo em um Cambissolo demonstrou otimizar o ganho em fósforo pelas plantas, quando o silício foi aplicado no solo numa etapa posterior à adubação fosfatada (CARVALHO, 1999). Fortes (1993), utilizando escórias de alto forno, obteve maior produção de matéria seca para o sorgo. Resultado semelhante foi observado por Lousada (1987), trabalhando com escórias em cultivos de sorgo e soja.

Porém, existe também a necessidade de reposição de outros elementos essenciais, tais como $\mathrm{Ca}, \mathrm{Mg}$, $\mathrm{K}$, de forma a melhorar a disponibilidade (VELOSO et al., 1992; KAMINSKI, 2000; ABREU; MURAOKA; OLIVEIRA, 2001). Esta reposição pode ser efetuada pela utilização de corretivos da acidez, o que auxilia também na diminuição do problema da adsorção de $\mathrm{P}$ pelos solos, pois com a elevação do $\mathrm{pH}$, ocorre um aumento da solubilidade dos fosfatos de ferro e alumínio, como minerais, aumento da concentração de $\mathrm{OH}^{-}$na solução do solo por troca de ligantes, reduzindo a adsorção do fosfato na fase sólida do solo (LEAL; VELLOSO, 1973; VASCONCELLOS et al., 1975; RAIJ, 1991; CASAGRANDE; CAMARGO, 1997; KAMINSK, 1983; ALVARADO; CAJUSTE, 1993). A combinação de uma fonte de potássio com calcário por Dias e Fioretto (2002), resultou em uma mistura denominada CALPOT. Os teores de $\mathrm{Ca}, \mathrm{Mg}$ e $\mathrm{K}$ da mistura eram determinados através da necessidade de reposição do solo, após verificação das análises químicas, sempre para atingir $50 \%$ de $\mathrm{Ca}^{+2}, 15 \%$ de $\mathrm{Mg}^{+2}$ e $3 \%$ de $\mathrm{K}^{+}$no complexo sortivo. Carneiro (2003) testou o CALPOT juntamente com silício e o fósforo para proporcionar a correção da fertilidade e a melhora da disponibilidade de fósforo, obtendo uma maior disponibilidade de fósforo no tratamento onde o CALPOT foi co-aplicado com silício e fósforo.

O objetivo deste trabalho visou melhorar o fornecimento dos elementos $\mathrm{Ca}, \mathrm{Mg}$ e $\mathrm{K}$ e a disponibilidade do fósforo para plantas de soja (Glycine max L.) em um Latossolo Vermelho Distroférrico em casa de vegetação, através da coaplicação da mistura CALPOT, silício e fósforo no solo.

\section{Material e Métodos}

Utilizou-se um Latossolo Vermelho Distroférrico coletado na Fazenda Escola da Universidade Estadual de Londrina - UEL - Londrina-PR, $\left(23^{\circ} 19^{\prime} \mathrm{S} ; 51^{\circ}\right.$ $11^{\prime} \mathrm{W}$ ) coletado na profundidade entre $60-70 \mathrm{~cm}$. O material foi seco ao ar, (TFSA) e passado em peneira de $2 \mathrm{~mm}$ (ABNT10) e submetido às análises físicas e químicas (EMPRESA BRASILEIRA DE PESQUISA AGROPECUÁRIA, 1997). O silício e a capacidade máxima de adsorção de fósforo (CMAP) foram determinados em (CARNEIRO, 2003) Tabela 1. 
Tabela 1. Atributos químicos e físicos do Latossolo Vermelho Distroférrico utilizado no experimento.

\begin{tabular}{|c|c|}
\hline ATRIBUTOS & SOLO \\
\hline pH em água & 5,3 \\
\hline $\mathrm{pH}$ em $\mathrm{CaCl}_{2}$ & 5,1 \\
\hline $\mathrm{P}\left(\mathrm{mg} \mathrm{dm}^{-3}\right)^{1 /}$ & 0,91 \\
\hline $\mathrm{K}\left(\mathrm{cmol}_{\mathrm{c}} \mathrm{dm}^{-3}\right)$ & 0,03 \\
\hline $\mathrm{Ca}\left(\mathrm{cmol}_{\mathrm{c}} \mathrm{dm}^{-3}\right)$ & 2,30 \\
\hline $\mathrm{Mg}\left(\mathrm{cmol}_{\mathrm{c}} \mathrm{dm}^{-3}\right)$ & 0,31 \\
\hline $\mathrm{Al}\left(\mathrm{cmol}_{\mathrm{c}} \mathrm{dm}^{-3}\right)$ & 0,20 \\
\hline $\mathrm{H}^{+}\left(\mathrm{cmol}_{\mathrm{c}} \mathrm{dm}^{-3}\right)$ & 4,41 \\
\hline $\mathrm{H}+\mathrm{Al}\left(\mathrm{cmol}_{\mathrm{c}} \mathrm{dm}^{-3}\right)$ & 4,61 \\
\hline $\mathrm{CO}\left(\mathrm{g} \mathrm{kg}^{-1}\right)^{2 /}$ & 4,82 \\
\hline $\mathrm{V} \%$ & 36,38 \\
\hline $\mathrm{CTC}_{\mathrm{pH} 7}\left(\mathrm{cmol}_{\mathrm{c}} \mathrm{dm}^{-3}\right)$ & 7,24 \\
\hline $\mathrm{S}\left(\mathrm{SO}^{-2}{ }_{4}\right)\left(\mathrm{mg} \mathrm{dm}^{-3}\right)$ & 80,55 \\
\hline $\operatorname{Mn}\left(\mathrm{mg} \mathrm{dm}{ }^{-3}\right)$ & 15,60 \\
\hline $\mathrm{Fe}\left(\mathrm{mg} \mathrm{dm}^{-3}\right)$ & 38,0 \\
\hline $\mathrm{Cu}\left(\mathrm{mg} \mathrm{dm}^{-3}\right)$ & 14,45 \\
\hline $\mathrm{Zn}\left(\mathrm{mg} \mathrm{dm}^{-3}\right)$ & 0,46 \\
\hline $\mathrm{B}\left(\mathrm{mg} \mathrm{dm}^{-3}\right)$ & 0,05 \\
\hline $\mathrm{Fe}_{2} \mathrm{O}_{3}\left(\mathrm{~g} \mathrm{~kg}^{-1}\right)^{3 /}$ & 266 \\
\hline $\mathrm{Al}_{2} \mathrm{O}_{3}\left(\mathrm{~g} \mathrm{~kg}^{-1}\right)^{3 /}$ & 177,5 \\
\hline $\mathrm{SiO}_{2}\left(\mathrm{~g} \mathrm{~kg}^{-1}\right)^{3 /}$ & 94,9 \\
\hline $\mathrm{CMAF}\left(\mathrm{mg} \mathrm{kg}^{-1}\right)^{4 /}$ & 3000 \\
\hline Si solúvel em água $\left(\mathrm{mg} \mathrm{dm}^{-3}\right)$ & 18,03 \\
\hline Si solúvel em ácido $\left(\mathrm{mg} \mathrm{dm}^{-3}\right)$ & 14,09 \\
\hline Areia $\left(\mathrm{g} \mathrm{kg}^{-1}\right)$ & 7,2 \\
\hline Silte $\left(\mathrm{g} \mathrm{kg}^{-1}\right)$ & 143,6 \\
\hline Argila $\left(\mathrm{g} \mathrm{kg}^{-1}\right)$ & 849,2 \\
\hline
\end{tabular}

${ }^{1 / E x t r a t o r ~ d e ~ P, ~ M e h l i c h-I ; ~}{ }^{2 /} \mathrm{CO}=$ carbono orgânico; ${ }^{3 /}$ oxidos do ataque sulfúrico (EMPRESA BRASILEIRA DE PESQUISA AGROPECUÁRIA, 1997); ${ }^{4 /} \mathrm{CMAF}=$ capacidade máxima de adsorção de fósforo.

O experimento foi conduzido em casa de vegetação com sete tratamentos e cinco repetições. Cada tratamento, após, misturado ao solo foi incubado em recipiente aberto, durante 60 dias à temperatura ambiente e $80 \%$ de umidade. Os tratamentos constaram de: 1) testemunha; 2) CALPOT $+\mathrm{Si}+\mathrm{P}$; 3) $\mathrm{CALPOT}+\mathrm{P}$; 4) CALPOT + $\mathrm{Si}$; 5) $\mathrm{Si}+\mathrm{P}$; 6) Calcário; 7) CALPOT. A dose $\mathrm{Ca}, \mathrm{Mg}$ e K como CALPOT aplicada foi estabelecida para se atingir um índice de saturação de $63 \%$, sendo $50 \%$ de Ca, $10 \%$ de $\mathrm{Mg}$ e $3 \%$ de $\mathrm{K}$, como definido no estudo de adsorção em (CARNEIRO, 2003). A dose de P foi
$100 \mathrm{~kg}$ de $\mathrm{P}$ por ha ${ }^{-1}$ de solo, utilizando como fonte de fósforo um resíduo industrial, denominado Fosfato Decantado - RLT2 tendo 20\% de $\mathrm{P}_{2} \mathrm{O}_{5}$ solúvel em CNA, $20 \%$ de $\mathrm{Ca}$ e $0,7 \%$ de $\mathrm{S}$. A dose de $\mathrm{Si}$ foi duas vezes a dose de $\mathrm{P},(200 \mathrm{~kg}$ de $\mathrm{Si}$ por ha-1 de solo). O silício utilizado (escória de siderurgia) foi fornecido pela Silifértil Ambiental Ltda (Tabela 2). Todos os elementos foram passados em peneira com abertura 0,3 mm (ABNT 50). O solo foi amostrado após incubação, para a realização das análises de pH, $\mathrm{P}$, $\mathrm{Al}, \mathrm{Ca}, \mathrm{Mg}$ e K (EMPRESA BRASILEIRA DE PESQUISA AGROPECUÁRIA, 1997). 
Carneiro, C. E. A. et al.

Tabela 2. Resultado médio das análises químicas (teores totais) da escória silicatada de siderurgia utilizada como fonte de silício.

\begin{tabular}{cccccc|ccccccc}
\hline \multicolumn{11}{c}{ Elementos Químicos } \\
\hline \multicolumn{11}{c}{ Macronutrientes $\left(\mathrm{g} \mathrm{kg}^{-1}\right)$} & & \multicolumn{7}{c}{ Micronutrientes $\left(\mathrm{mg} \mathrm{kg}^{-1}\right)$} \\
\hline $\mathrm{Ca}$ & $\mathrm{Mg}$ & $\mathrm{K}$ & $\mathrm{P}$ & $\mathrm{S}$ & $\mathrm{Si}$ & $\mathrm{Zn}$ & $\mathrm{Fe}$ & $\mathrm{Mn}$ & $\mathrm{Cu}$ & $\mathrm{B}$ & $\mathrm{Mo}$ & $\mathrm{Co}$ \\
\hline 400 & 90 & 9 & 10 & 9 & 420 & 700 & 16 & 29 & 300 & 150 & 100 & 70 \\
\hline
\end{tabular}

O solo incubado com cada tratamento foi colocado em vaso de plástico com capacidade máxima de 4 $\mathrm{kg}$, recebendo, cada um, $3 \mathrm{~kg}$ de solo, equivalendo a uma densidade de $1,2 \mathrm{~kg} \mathrm{dm}^{-3}$. Utilizou-se como planta indicadora a soja, (Glycine max L.), cultivar BRS 133, cujas sementes foram cedidas pela EmbrapaSoja. Foram semeadas quatro sementes por vaso, deixando duas plantas por vaso após desbaste. Os vasos foram irrigados em turnos de rega para manter a umidade do solo a $70 \%$ do volume de poros.

Aos 40 dias após a semeadura, as plantas foram cortadas rente ao solo, separadas em raiz, caule e folha e secas a $65^{\circ} \mathrm{C}$ até peso constante, em estufa com ventilação, em seguida avaliou-se matéria seca das raízes e parte aérea. Para as análises químicas, os materiais vegetais foram moídos e peneirados, determinando-se macro e micro nutrientes. Os resultados foram submetidos à análise de variância e as médias comparadas pelo teste de Tukey a 5\% de probabilidade de erro.

\section{Resultados e Discussão}

Os resultados do experimento revelaram as alterações a que foram submetidos os elementos $\mathrm{Ca}$, $\mathrm{Mg}$ e K do complexo sortivo (Tabela 3) do solo de acordo com Carneiro (2003), onde a co-aplicação dos elementos possibilitou o estabelecimento do equilíbrio químico para os elementos estudados nos determinados teores e aumentou a disponibilidade de fósforo no solo.

Tabela 3. Saturação do complexo de troca do solo por cátions básicos e $\mathrm{Al}^{+3}$, fósforo e $\mathrm{pH}$ após incubação de 60 dias com os respectivos tratamentos.

\begin{tabular}{cccccccc}
\hline & \multicolumn{5}{c}{ Elementos $(\%)$} & & $\mathrm{P}$ \\
\cline { 2 - 4 } Tratamentos & $\mathrm{K}^{+}$ & $\mathrm{Ca}^{+2}$ & $\mathrm{Mg}^{+2}$ & $\mathrm{Al}^{+3}$ & & $\left(\mathrm{mg} \mathrm{dm}^{-3}\right)$ & $\mathrm{pH}$ \\
\hline TESTEMUNHA & $0,41 \mathrm{D}$ & $32,00 \mathrm{G}$ & $3,23 \mathrm{E}$ & $13,52 \mathrm{~A}$ & & $0,89 \mathrm{D}$ & $5,3 \mathrm{~F}$ \\
CALPOT + P + Si & $3,59 \mathrm{~B}$ & $66,57 \mathrm{~A}$ & $14,05 \mathrm{~A}$ & $0,35 \mathrm{C}$ & & $15,62 \mathrm{~A}$ & $6,4 \mathrm{~B}$ \\
CALPOT + P & $3,86 \mathrm{~A}$ & $62,01 \mathrm{~B}$ & $12,92 \mathrm{~B}$ & $0,00 \mathrm{D}$ & & $9,90 \mathrm{~B}$ & $6,1 \mathrm{D}$ \\
CALPOT + Si & $3,59 \mathrm{~B}$ & $58,14 \mathrm{D}$ & $13,81 \mathrm{~A}$ & $0,35 \mathrm{C}$ & & $1,03 \mathrm{D}$ & $6,4 \mathrm{~B}$ \\
$\mathrm{Si}+\mathrm{P}$ & $0,69 \mathrm{C}$ & $53,72 \mathrm{~F}$ & $9,94 \mathrm{D}$ & $2,46 \mathrm{~B}$ & & $6,64 \mathrm{C}$ & $5,7 \mathrm{E}$ \\
CALCÁRIO & $0,41 \mathrm{D}$ & $56,35 \mathrm{E}$ & $11,98 \mathrm{C}$ & $0,35 \mathrm{C}$ & & $0,92 \mathrm{D}$ & $6,5 \mathrm{~A}$ \\
CALPOT & $3,86 \mathrm{~A}$ & $60,08 \mathrm{C}$ & $13,36 \mathrm{AB}$ & $0,35 \mathrm{C}$ & & $0,92 \mathrm{D}$ & $6,3 \mathrm{C}$ \\
\hline C.V.(\%) & 0 & 1,33 & 3,06 & 4,79 & & 5,95 & 0,33 \\
\hline D.M.S. & 0,26 & 1,48 & 0,69 & 0,23 & & 0,613 & 0,041 \\
\hline
\end{tabular}

Médias com letras distintas, na coluna, diferem entre si pelo teste de Tukey a 5\% de probabilidade de erro.

O crescimento da soja é representado pelas médias do peso do sistema radicular, de caule, de folhas e de matéria seca total (Tabela 4). Quando existe um equilíbrio entre os nutrientes aplicados (baseado no equilíbrio proposto), a planta responde positivamente em crescimento o que pode ser visto no tratamento CALPOT $+\mathrm{P}+\mathrm{Si}$ com maior valor na matéria seca total, $2 \mathrm{~g}_{\text {planta }}{ }^{-1}$. Nos tratamentos $\mathrm{CALPOT}+\mathrm{P}$ e CALPOT $+\mathrm{Si}$, o peso de raiz não diferiu 0,47 g planta $^{-1}$, nestes tratamentos, foram 
aplicados os mesmos cátions, e apresentavam a proposta do equilíbrio químico dos elementos, mudando apenas o fornecimento de entre P e Si. Com base nos dados observou-se que a aplicação de silício influenciou o aumento do desenvolvimento da raiz do mesmo modo que o fósforo, onde os tratamentos CALPOT + P e CALPOT + Si não diferem. A utilização do Si como fonte na nutrição da planta vem crescendo a cada dia e sua utilização tem mostradose promissora quanto a redução na incidência de doenças e aumento na produção (KORNDÖFER, 2002). Segundo Korndöfer (2002) os efeitos benéficos da aplicação de silicatos de Ca e Mg estão, normalmente, associados ao aumento na disponibilidade do Si, à elevação do pH e ao aumento do $\mathrm{Ca}$ e $\mathrm{Mg}$ trocável do solo. No tratamento CALPOT ocorreu menor produção de matéria seca devido a falta de fornecimento do fósforo, elemento essencial ao metabolismo da planta. Já para o tratamento Calcário a menor produção de matéria seca pode ser explicada pela falta do fornecimento do K, o que também pode ter acarretado o desbalanço das bases no solo, dificultando a absorção das mesmas e a falta do fornecimento de fósforo. Lembrando que o mesmo encontra-se em nível bastante baixo, o que pode ser verificado na Testemunha, $\left(0,89 \mathrm{mg} \mathrm{kg}^{-1}\right) \mathrm{e}$ a disponibilidade se deu através do fornecimento.

Tabela 4. Matéria seca de raíz, caule, folhas e total das plantas de soja (g planta $\left.{ }^{-1}\right)$.

\begin{tabular}{ccccc}
\hline Tratamentos & Raízes & Caule & Folhas & Mat.Seca Total \\
\hline TESTEMUNHA & $0,39 \mathrm{BC}$ & $0,41 \mathrm{D}$ & $0,37 \mathrm{C}$ & $1,17 \mathrm{D}$ \\
CALPOT + P + Si & $0,53 \mathrm{~A}$ & $0,75 \mathrm{~A}$ & $0,71 \mathrm{~A}$ & $2,00 \mathrm{~A}$ \\
CALPOT + P & $0,47 \mathrm{AB}$ & $0,62 \mathrm{AB}$ & $0,57 \mathrm{~B}$ & $1,66 \mathrm{~B}$ \\
CALPOT + Si & $0,47 \mathrm{AB}$ & $0,51 \mathrm{BCD}$ & $0,46 \mathrm{BC}$ & $1,45 \mathrm{BCD}$ \\
$\mathrm{Si}+\mathrm{P}$ & $0,39 \mathrm{BC}$ & $0,56 \mathrm{BC}$ & $0,56 \mathrm{~B}$ & $1,52 \mathrm{BC}$ \\
CALCÁRIO & $0,35 \mathrm{C}$ & $0,45 \mathrm{CD}$ & $0,42 \mathrm{C}$ & $1,24 \mathrm{CD}$ \\
CALPOT & $0,38 \mathrm{BC}$ & $0,47 \mathrm{CD}$ & $0,44 \mathrm{C}$ & $1,30 \mathrm{CD}$ \\
C.V.\% & 13,69 & 12,24 & 10,87 & 11,27 \\
DMS & 0,11 & 0,133 & 0,11 & 0,33 \\
\hline
\end{tabular}

Médias com letras distintas, na coluna, diferem entre si pelo teste de Tukey a 5\% de probabilidade de erro.

A aplicação do tratamento CALPOT $+\mathrm{P}+\mathrm{Si}$, proporcionou o maior desenvolvimento das plantas em termos de peso seco de folhas e de matéria seca total (Tabela 4). As plantas responderam positivamente à associação dos elementos no tratamento CALPOT $+\mathrm{P}+\mathrm{Si}$, tendo $17 \%$ mais matéria seca total que no tratamento CALPOT $+\mathrm{P}$, que utiliza os elementos convencionalmente aplicados na agricultura, ou seja, aplicação de calcário, potássio e fósforo.

As maiores absorções de fósforo pela soja foram nos tratamentos CALPOT $+\mathrm{P}+\mathrm{Si}$, CALPOT $+\mathrm{P}, \mathrm{P}$ $+\mathrm{Si}$ (Tabela 4) os quais também apresentaram maiores teores de fósforo no solo. De acordo com Prado e
Fernandes (2000) a escória proporciona um aumento do P disponível no solo, deve-se este efeito a saturação dos sítios de adsorção de fósforo pelo silício. Em todos os tratamentos que foi aplicado K, houve resposta positiva da planta, não há diferença (Tabela 4), isto indica que a co-aplicação na forma de CALPOT ou CALPOT $+\mathrm{P}$, CALPOT $+\mathrm{Si}$ ou CALPOT + P + Si não influencia de forma negativa a absorção de K pela planta. Mascarenhas (2000) conclui que as maiores produtividades de grãos de soja foram obtidas quando a concentração de potássio nas folhas variaram de 13 a $17 \mathrm{~g} \mathrm{~kg}^{-1}$, observando a tabela 5 pode-se ver que o potássio na planta para os tratamentos, supera o teor obtido por Mascarenhas. 
Apesar do cálcio não ter diferido estatisticamente observa-se que o maior teor nas folhas foi para o tratamento CALPOT $+\mathrm{P}+\mathrm{Si}$. Observando teores de $\mathrm{Ca}$, é possível inferir que existe a necessidade de que os elementos estejam em equilíbrio no solo para que ocorra uma melhor nutrição, onde o tratamento CALPOT $+\mathrm{P}+$ Si representa o melhor equilíbrio.

Tabela 5. Conteúdo de macro-elementos nas folhas da soja coletadas aos 40 dias após semeadura em Latossolo Vermelho Distroférrico submetido aos tratamentos.

\begin{tabular}{cccccc}
\hline Tratamentos & \multicolumn{5}{c}{$\left(\mathrm{g} \mathrm{kg}^{-1}\right)$} \\
\cline { 2 - 6 } & $\mathrm{N}$ & $\mathrm{P}$ & $\mathrm{K}^{+}$ & $\mathrm{Ca}^{2+}$ & $\mathrm{Mg}^{2+}$ \\
\hline TESTEMUNHA & $31,08 \mathrm{AB}$ & $1,3 \mathrm{C}$ & $8,43 \mathrm{~B}$ & 12,70 & $4,81 \mathrm{C}$ \\
CALPOT + P + Si & $35,3 \mathrm{~A}$ & $2,1 \mathrm{~A}$ & $24,4 \mathrm{~A}$ & 19,4 & $6,0 \mathrm{AB}$ \\
CALPOT + P & $31,2 \mathrm{AB}$ & $1,8 \mathrm{~A}$ & $21,0 \mathrm{~A}$ & 16,3 & $5,1 \mathrm{ABC}$ \\
CALPOT + Si & $30,5 \mathrm{AB}$ & $1,3 \mathrm{C}$ & $23,3 \mathrm{~A}$ & 17,0 & $5,1 \mathrm{ABC}$ \\
Si + P & $26,1 \mathrm{~B}$ & $1,7 \mathrm{AB}$ & $8,4 \mathrm{~B}$ & 18,8 & $6,4 \mathrm{AB}$ \\
CALCÁRIO & $29,0 \mathrm{AB}$ & $1,3 \mathrm{C}$ & $8,2 \mathrm{~B}$ & 18,4 & $6,8 \mathrm{~A}$ \\
CALPOT & $26,7 \mathrm{~B}$ & $1,4 \mathrm{BC}$ & $20,5 \mathrm{~A}$ & 14,0 & $4,1 \mathrm{C}$ \\
\hline C.V.(\%) & 13,7 & 13,5 & 16,0 & 24,2 & 16,5 \\
\hline D.M.S. & 8,2 & 0,4 & 5,2 & 8,1 & 1,8 \\
\hline
\end{tabular}

Médias com letras distintas, na coluna, diferem entre si pelo teste de Tukey a 5\% de probabilidade de erro.

Os conteúdos de Fe e Zn nas folhas de soja não diferiram estatisticamente entre os tratamentos, indicativo de que a mistura do tratamento CALPOT + $\mathrm{P}+$ Si não alterou a absorção destes elementos. O cobre, elemento essencial na síntese de compostos que participam do metabolismo, está abaixo do nível de interpretação $\left(10 \mathrm{mg} \mathrm{kg}^{-1}\right)$ para todos os tratamentos, isto ocorreu devido a não aplicação do elemento. Maior absorção de Mn pela planta, se deu na testemunha (Tabela 6), onde o pH do solo foi de 5,3 (Tabela 3). A disponibilidade de Mn no solo é controlada principalmente pelo $\mathrm{pH}$, e pelo potencial de oxidação dos solos (LINDSAY, 1982). Na medida que houve aumento de $\mathrm{pH}$, ocorreu menor absorção do Mn.

Tabela 6. Conteúdo de micro-elementos nas folhas da soja coletadas aos 40 dias após semeadura em Latossolo Vermelho Distroférrico submetido aos tratamentos.

\begin{tabular}{ccccc}
\hline Tratamentos & \multicolumn{3}{c}{$\left(\mathrm{mg} \mathrm{kg}^{-1}\right)$} & $\mathrm{Zn}^{2+}$ \\
\cline { 2 - 5 } & $\mathrm{Cu}^{2+}$ & $\mathrm{Fe}^{2+}$ & $502,61 \mathrm{~A}$ & $71,47 \mathrm{~A}$ \\
\hline TESTEMUNHA & $7,14 \mathrm{AB}$ & $154,18 \mathrm{~A}$ & $370,45 \mathrm{~B}$ & $71,48 \mathrm{~A}$ \\
CALPOT + P + Si & $9,18 \mathrm{~A}$ & $177,35 \mathrm{~A}$ & $329,96 \mathrm{BCD}$ & $5,29 \mathrm{~A}$ \\
CALPOT + P & $7,30 \mathrm{AB}$ & $194,49 \mathrm{~A}$ & $26,71 \mathrm{~A}$ \\
CALPOT + Si & $5,13 \mathrm{~B}$ & $262,13 \mathrm{~A}$ & $289,76 \mathrm{BCD}$ & $72,27 \mathrm{~A}$ \\
Si + P & $5,77 \mathrm{AB}$ & $194,78 \mathrm{~A}$ & $342,34 \mathrm{BC}$ & $55,91 \mathrm{~A}$ \\
CALCÁRIO & $7,31 \mathrm{AB}$ & $178,57 \mathrm{~A}$ & $230,54 \mathrm{D}$ & $53,92 \mathrm{~A}$ \\
CALPOT & $6,96 \mathrm{AB}$ & $204,13 \mathrm{~A}$ & $243,85 \mathrm{D}$ & 24,80 \\
\hline C.V.( $\%)$ & 26,00 & 42,50 & 16,38 & 30,36 \\
\hline D.M.S. & 3,63 & 166,38 & 108,45 & \\
\hline
\end{tabular}

Médias com letras distintas, na coluna, diferem entre si pelo teste de Tukey a 5\% de probabilidade de erro. 


\section{Conclusão}

As plantas de soja responderam de forma positiva à co-aplicação destes elementos no solo, resultando em maior peso da matéria seca total.

A co-aplicação de $\mathrm{Ca}, \mathrm{Mg}, \mathrm{K}, \mathrm{P}, \mathrm{Si}$, não limita o fornecimento de nenhum nutriente testado e ainda aumenta a disponibilidade de fósforo no solo, o que possibilita melhor nutrição da soja.

\section{Referências}

ABREU JR., C. H.; MURAOKA, T.; OLIVEIRA, F. C. Cátions trocáveis, capacidade de troca de cátions e saturação por bases em solos brasileiros adubados com composto de lixo urbano. Scientia Agrícola., Piracicaba, v.58,n.4,p.813-824, 2001.

ALVARADO, J.; CAJUSTE, L. J. Encalado y retención de fósforo en suelos derivados de cenizas volcánicas. Turrialba, San Jose, v.43, n.4, p.235-241, 1993.

CARNEIRO, C. E. A. Disponibilidade de fósforo no solo pela interação do CALPOT e silício. 2003. Dissertação (Mestrado em Agronomia) - Universidade Estadual de Londrina, Londrina.

CARVALHO, R. Interações silício-fósforo em latossolo vermelho-escuro e cambissolo cultivados com mudas de eucalipto. 1999. Tese (Doutorado) - Universidade Federal de Lavras, Lavras.

CASAGRANDE, J. C.; CAMARGO, O. A. Adsorção de fosfato em solos com caráter ácrico avaliada por um modelo de complexação de superfície. Revista Brasileira Ciência do Solo, Viçosa, v.21, p.353-360, 1997.

DIAS, A. M.; FIORETTO, R. A. Utilização do efluente da industrialização de trifuralina como fonte de potássio no solo. Londrina: Universidade Estadual de Londrina, 2002. (Informe Técnico, 2).

EMPRESA BRASILEIRA DE PESQUISA AGROPECUÁRIA. Manual de métodos de análise de solos. 2 ed. Rio de Janeiro: Centro Nacional de Pesquisa de Solos, 1997.

FORTES, J.L.O. Eficiência de duas escórias de siderurgia, do Estado do Maranhão, na correção da acidez do solo. 1993. Dissertação (Mestrado) - Universidade Federal de Viçosa, Viçosa.
KAMINSKI, J. Efeito de cinco fosfatos pré-, co- e pósaplicados ao calcário no suprimento de fósforo ao sorgo em três solos ácidos. 1983. Tese (Doutorado em Agronomia) - Escola Superior de Agricultura Luiz de Queiroz, Piracicaba.

KAMINSKI, J. Uso de corretivos da acidez do solo no plantio direto. Pelotas: Núcleo Regional Sul, Sociedade Brasileira de Ciência do Solo, 2000. (Boletim Técnico n. 4)

KORNDÖRFER, G. H.; PEREIRA, H. S.; CAMARGO, M. S. Silicatos de cálcio e magnésio na agricultura. Uberlândia, 2002. (Boletim Técnico n.1).

LEAL, J. R.; VELLOSO, A C. X. Dessorção do fosfato adsorvido em latossolos sob vegetação de cerrado II. Reversibilidade da isoterma de adsorção de fosfato em relação ao $\mathrm{pH}$ da solução de equilíbrio. Pesquisa Agropecuária Brasileira, Brasília, v.8, p.89-92, 1973.

LEITE, P. C. Interação silício-fósforo em latossolo roxo cultivado com sorgo em casa de vegetação. 1997. Tese (Doutorado em Solos e Nutrição de Plantas) - Universidade Federal de Viçosa, Viçosa.

LINDSAY, W. L. Chemical equilibrio in soils. New York: John W. \& Sons, 1982,

LOUSADA, P. T. C. Eficiência de uma escória de siderurgia como corretivo e fertilizante do solo. 1987. Dissertação (Mestrado em solos e nutrição de plantas) Universidade Federal de Viçosa, Viçosa.

MASCARENHAS, H. A. A.; TANAKA, R. T.; CARMELLO, Q. A. C.; Gallo, P. B; AMBROSANO, G. M. B. Calcário e potássio para a cultura de soja. Scientia Agricola, Piracicaba, v.57, n.3, p.445-449, 2000.

MOTTA, P. E. F.; CURI, N.; SIQUEIRA, J. O. VAN RAIJ, B. FURITINI, A. E.; LIMA, J. M. Adsorção e formas de fósforo em latossolos: influência da minarelogia e histórico de uso. Revista Brasileira Ciência do Solo, Viçosa, v.26, p.349-359, 2002.

MUNIZ, A. S.; NOVAIS, R. F.; BARROS, N. F.; NEVES J. C. L. Nível crítico de fósforo na parte aérea da soja como variável do fator capacidade de fósforo do solo. Revista Brasileira Ciência do Solo, Campinas, v.9, p.237-243, 1985.

PARFITT, R. L. Anion adsorption by soils and soil materials. Advances in Agronomy, Orlando, v.30, p.1-50, 1978.

PRADO, R. M.; FERNANDES, F.M. Escória de siderurgia e calcário na correção da acidez do solo cultivado com cana-de-açúcar em vaso. Scientia Agricola, Piracicaba, v.57, n.4, p.739-744, 2000. 
RAIJ, B. van. Fertilidade do solo e adubação. São Paulo: Agronômica Ceres, 1991.

SILVA, E. B.; NOGUEIRA,F.D.; GUIMARÃES, P. T. G. Uso do DRIS na avaliação do estado nutricional do cafeeiro em resposta à adubação potássica. Revista Brasileira Ciência do Solo, Viçosa, v.27, n.2, p.247-255, 2003.

VASCONCELOS, C. A.; BRAGA, J. M.; NOVAIS, R. F.; PINTO, O. C. B. Fósforo em dois latossolos do Estado de MG. III - Relações entre planta, solo e P. Revista Ceres, Viçosa, v.22, n.119, p.22-49, 1975.
VELOSO, C. A.C.; BORGES, A. L.; MUNIZ, A. S.; VEIGAS, I. A. J. M. Efeito de diferentes materiais no $\mathrm{pH}$ do solo. Scientia Agrícola, Piracicaba, v.49, n.1, p.123-128, 1992.

VOLKWEISS, S. J.; RAIJ, B. van. Retenção e disponibilidade de fósforo em solos. In: SIMPÓSIO SOBRE CERRADO: BASE PARA A UTILIZAÇÃO AGROPECUARIA. 4, 1976, Brasília. Anais.. São Paulo: EDUSP, 1977. p.317-332. 